\title{
Young and Old Subsystems of Flare Stars
}

\author{
L.V. Mirzoyan, V.V. Hambarian \\ Byurakan Astrophysical Observatory, Armenia
}

Photographic observations with wide-field telescopes of flare stars in the Orion association and the Pleiades cluster have been carried out since 40 years. About 500 flare stars were found in each of these systems. The ages of these stellar systems differ by one or two orders of magnitude (Mirzoyan 1991). This fact allows us to compare them, in order to show that they fit into the concept, suggested first by Haro (1957), that the evolutionary status of flare stars is a stage of red dwarf star evolution which follows that of the T Tau stars (Haro 1976, Ambartsumian \& Mirzoyan 1970).

The basic difference between the Orion and Pleiades subsystems of flare stars has been known for a long time: the coexistence of flare stars and $T$ Tau stars in the Orion association, and the absence of the latter group in the Pleiades. Some of the T Tau stars show flare activity (Haro 1964). This fact and the existence of multiple systems of trapezium type, which are dynamically unstable, in the Orion association, are arguments in favour of an evolutionary connection between these two types of stars.

Another noteworthy difference between the Orion and Pleiades subsystems is the luminosity of the brightest flare stars, which is $3^{\mathrm{m}}$ brighter in Orion. For flare stars of the same luminosity, the flare activity is higher in Orion.

The distribution of the mean flare frequencies is also different for the Orion and Pleiades subsystems, which can be explained by the difference of their luminosity functions. The reason is that the mean flare frequency increases towards lower luminosities.

All abovementioned differences between the flare star subsystems of different ages are in remarkable accordance with the evolutionary status of flare stars. For example, the absence of $\mathrm{T}$ Tau stars in the middle-age Pleiades cluster is naturally explained by the young age of $\mathrm{T}$ Tau stars. They belong to the characteristic population of stellar associations (Ambartsumian 1947) and are transformed later into flare stars (Mirzoyan 1981). The higher luminosity of the brightest flaring stars in the younger subsystem is a consequence of the fact that the rate of evolution increases with mass (or luminosity).

It should be added that the subsystem of flare stars in Orion is much larger than that of the Pleiades. However, the estimated total number of flare stars in the Orion association is only two times larger than in the Pleiades cluster. It 
seems that this difference is a consequence of observational selection caused by the noticeable difference in distance. The total number of flare stars in Orion must be larger than that in the Pleiades by at least one order of magnitude.

\section{References}

Ambartsumian V.A., 1947, Stellar Evolution and Astrophysics, Acad. Sci. of Armenia, Yerevan

Ambartsumian V.A., Mirzoyan L.V., 1970, in IAU Coll. No. 15, New Directions and New Frontiers in Variable Star Research, Veröff. Bamberg 9, No. 100, p. 98

Haro G., 1957, in IAU Symp. No. 3, Non-Stable Stars, G.H. Herbig (ed.), Cambridge University Press, Cambridge, p. 26

Haro G., 1964, in IAU-URSI Symp. No. 20, The Galaxy and the Magellanic Clouds, F.J. Kerr and A.W. Rodgers (eds.), Australian Acad. Sci., Canberra, p. 30

Haro G., 1976, Bol. Inst. Tonantzintla 2, 3

Mirzoyan L.V., 1981, Stellar Instability and Evolution, Acad. Sci. of Armenia, Yerevan Mirzoyan L.V., 1991, Early Stages of Stellar Evolution, Acad. Sci. of Armenia, Yerevan 